\title{
Mit Sicherheit zu etwas Neuem: Zur Bedeutung psychologischer Sicherheit in der Post-Merger-Integration (PMI)
}

\author{
Alexander Pfurr · Freimut Schirrmacher
}

Eingegangen: 3. Mai 2021 / Angenommen: 17. Juni 2021 / Online publiziert: 3. August 2021

(C) Der/die Autor(en) 2021

Zusammenfassung Zahlreiche Unternehmensfusionen in Deutschland scheitern oder bleiben weit hinter den Erwartungen zurück. Besondere Bedeutung für das Gelingen von Fusionen hat die Gestaltung der Post-Merger-Integration, also die Phase des Zusammenführens der beiden vormals selbstständigen Unternehmen. Das von Amy C. Edmondson entwickelte Konzept der psychologischen Sicherheit könnte hierfür eine wichtige Funktion zur Orientierung und Handlungsleitung erhalten. Die erste Auswertung von Ergebnissen einer qualitativen Befragung von acht Post-Merger erfahrenen Beratungspersonen zeigt, dass dieses Konzept in Deutschland bislang noch nicht explizit eingesetzt wurde, aber dazu dienen kann, dass Firmenfusionen erfolgreicher abschließen. Interventionen und Maßnahmen, die hierbei zum Einsatz kommen können, sind gegenüber einer rein betriebswirtschaftlichen Denkart komplexer und machen die Begleitung durch externe Prozessberatende sinnvoll.

Schlüsselwörter Firmenfusionen · Psychologische Sicherheit · Post-MergerIntegration · Faktor Mensch

Dipl.-Oec. Alexander Pfurr, MBA $(\bowtie)$

ISABEA consult, Harleshäuser Str. 62, 34130 Kassel, Deutschland

E-Mail: apfurr@hotmail.com

Dipl.-Oec. Alexander Pfurr, MBA - Prof. Dr. Freimut Schirrmacher, M.A.

Institut für Psychologie, Universität Kassel, Kassel, Deutschland

E-Mail: freimut-schirrmacher@web.de

Prof. Dr. Freimut Schirrmacher, M.A.

Parkstraße 30, 34277 Fuldabrück, Deutschland 


\title{
With safety to something afresh: the importance of psychological safety in post merger integration (PMI)
}

\begin{abstract}
A large number of mergers in Germany fail or fall far short of expectations. The design process of post-merger integration, i.e. the phase of merging the two formerly independent companies, is of particular importance for the success of mergers in general. The concept of psychological safety developed by Amy C. Edmondson could play an important role in this process. The first analysis of the results of a qualitative survey of eight experienced consultants shows that this concept in Germany has not yet been explicitly taken into account. However, it could lay the foundations for companies to complete mergers more successfully. Interventions and measures that can be used in this regard are more complex than a purely businessoriented way of thinking and make it useful to be accompanied by external process consultants.
\end{abstract}

Keywords Mergers \& Acquisitions · Psychological Safety · Post-MergerIntegration · Human factor

\section{Einführung}

Unternehmensfusionen, -zusammenschlüsse und -übernahmen finden weltweit täglich statt. Größere Transaktionen werden von der Öffentlichkeit meist aufmerksam verfolgt. Zu denken ist dabei etwa an die erst kürzlich genehmigte Übernahme des Bahntechnikunternehmens Bombardier durch Alstom oder - etwas weiter zurück an die Fusion der Stahlgiganten Thyssen und Krupp im Jahr 1997.

Die aus den USA kommende Sammelbezeichnung „Mergers and Acquisitions“, abgekürzt „M\&A“, steht für eben diese Fusionen und Käufe und hat sich mittlerweile auch im deutschen Sprachraum durchgesetzt. Vielfach werden solche Prozesse von Unternehmensberatungen begleitet. Grundsätzlich lassen sich meist drei Phasen für M\&A-Prozesse unterscheiden: Vorbereitung, die eigentliche Transaktion und die Integration nach dem formalen Akt des Zusammenschlusses (vgl. Coenenberg et al. 2002, S. 179). In der Integrationsphase, die auch als „Post-Merger-Integration“, abgekürzt „PMI“, bezeichnet wird, geht es um die organisatorische und kulturelle Zusammenführung der beteiligten, vormals getrennten Unternehmensparteien.

So spektakulär derartige Prozesse auch von Presse und Öffentlichkeit wahrgenommen werden, so ernüchternd sind dann oft die Ergebnisse: Nur 1/3 der M\&AProzesse in Deutschland verlaufen letztlich erfolgreich und gelingen (Backovic et al. 2019). Vielfach bleiben die Unternehmenserfolge hinter den hoch gesteckten Erwartungen zurück. In nicht wenigen der vom Marketing zuvor als Erfolgsschlager gepriesenen Fusionen kommt es wieder zu Rückabwicklungen oder (Not-)Verkäufen von Unternehmensteilen, z. B. die Trennung von Daimler und Chrysler nach milliardenschweren Verlusten in der Fusionsphase. Solche Rückabwicklungen haben unterschiedliche geschäftliche Gründe, die im Einzelnen analysiert werden müssen. Solches Scheitern bzw. ein Zurückbleiben der prognostizierten Ergebnisse bei Fusionen könnten aber zu einem Teil in den M\&A-Prozessen selbst begründet liegen. 
Dies gilt insbesondere für die Integrationsphase. Diese stellt sich je nach Art des Zusammenschlusses und des jeweiligen Settings unterschiedlich dar: Ein erstes Szenario ist die feindliche Übernahme des einen Unternehmens durch ein anderes, also eine Absorptionsakquisition, in der eine komplette Konsolidierung aller Aktivitäten inklusive der unterschiedlichen Kulturen stattfinden soll. Ein zweites Szenario stellt der intendierte friedliche Zusammenschluss von zwei gleichberechtigten Firmen dar, der auch als „Merger of Equals“ bezeichnet wird. Hierbei kommt es meist zu einer sogenannten symbiotischen Akquisition, in der nach einer Gewöhnungsphase der Autonomiebedarf des akquirierten Unternehmens mit dem starken Integrationsbedarf des Erwerbers in Einklang gebracht werden muss. Ein drittes Szenario betrifft Erhaltungsakquisitionen, welche die „schwächste“ Form einer Integration darstellen. Hier soll das erworbene Unternehmen meist bei diagonalen, also geschäftsartfremden Zusammenschlüssen erhalten bleiben. Schon diese drei Szenarien lassen erkennbar werden, dass Unternehmensfusionen komplexe Vorhaben sind, die deshalb umgangssprachlich auch als „Königsdisziplin des Managements“ bezeichnet werden. Die Gründe für eine M\&A-Transaktion divergieren stark und haben in ihrer jeweiligen Ausprägung dann ebenfalls erhebliche Auswirkung auf psychosoziale Konstellationen und Entwicklungsspielräume der Integrationsphase.

Im Folgenden gehen wir der Fragestellung nach, ob und inwiefern das Scheitern solcher für Unternehmen überlebenswichtigen Prozesse auch in einer mangelnden Reflexivität und Handlungsführung psychosozialer Aspekte in den Post-Merger-Aktivitäten begründet liegen könnte, also in einer Schwäche bzw. sogar dem Ausfall einer wichtigen Säule des Prozessmanagements in dieser sensiblen Phase. Dabei ist klar: Wenn der (neuen) Unternehmensleitung, unterstützt durch die Methoden des HR-Managements, keine sukzessive unternehmenskulturelle Zusammenführung gelingt, wird sich dies auf den Integrationsprozess und auf die prognostizierten Fusionsergebnisse insgesamt negativ auswirken, bis hin zu einem möglichen Scheitern.

Festzuhalten ist aber auch: Nach einer vollzogenen Akquisition steht das Management unter besonderem Druck aller möglichen Stakeholder, also der Eigentümerseite, der Banken, der Mitarbeitenden, der Kunden und oft genug auch der Öffentlichkeit. Es muss nun unter diesem Druck beweisen, dass sich das zuvor formulierte Erwartungsprofil der Gründe für das Zusammengehen auch tatsächlich bestätigt: Synergieerwartungen, Rationalisierungs- und Kapitalisierungsmaßnahmen, Ergebniseffektivität, Wachstum, verbesserte Wettbewerbsstellung. Entsprechend müssen vom Management Mittel und Wege gefunden werden, um Produkte, Prozesse, Technologien und nicht zuletzt die Mitarbeiter/innen effektiv zusammenzuführen, damit die gemeinsamen unternehmerischen Ziele erreicht werden (vgl. Barnikel 2007, S. 16). Die Post-Merger-Integration ist also in besonderer Weise von strukturierten, zielgerichteten und lösungsorientierten Aktivitäten bzw. betriebswirtschaftlichen Prozessen und Perspektiven geprägt. Durch die fokussierte Ausrichtung auf diese kommerziellen und rechtlichen Herausforderungen wird zwar unter hohem Druck versucht, den an die M\&A-Transaktion gerichteten Erwartungen gerecht zu werden jedoch stellt sich gerade deshalb die Frage, wie es mit den ,,weichen“ Faktoren aussieht und wie diese überhaupt fassbar und in den Gesamtprozess integrierbar sind. Dies gilt auch für den aktiven Einbezug von Mitarbeitenden und Führungskräften in die Gestaltung solcher Prozesse (vgl. Berner 2017, S. 54). 
Amy C. Edmondson, Professorin an der Harvard Business School, hat mit ihren vielfältigen Studien und Publikationen den Begriff „Psychological Safety“ entwickelt und eingeführt. Im Folgenden wollen wir dieses Konzept in Bezug auf das spezifische Umfeld und die Herausforderungen des Post-Merger-Integrationsprozesses fokussieren. Danach werden wir mit einem Blick in die Expertenpraxis, d.h. mit Erfahrungen und Erfolgsfaktoren in der Gestaltung von Post-Merger-Prozessen fortfahren. Hierbei werden Ergebnisse aus acht Interviews, die im Rahmen einer Masterarbeit an der Universität Kassel geführt wurden, verarbeitet. Im Anschluss daran werden die aus der Auswertung gewonnenen Perspektiven aufgezeigt, um eine entsprechende Prozessreflexivität für die Post-Merger-Integration sichtbar zu machen.

\section{Das Konzept der „Psychologischen Sicherheit“" als Perspektive in der Post-Merger-Integration}

Im Jahr 2012 startete Google das „Aristoteles Projekt“: Zwei Jahre lang erforschte der US-amerikanische Konzern unter der Leitung von Julia Rozovsky mit Unterstützung von Statistikern, Psychologen, Soziologen und Technikern 180 Teams aus der eigenen Organisation, um herauszufinden, welche Kriterien für die beste Leistung im Team zusammentreffen müssen. Auf allen Hierarchieebenen wurden Interviews geführt. Je nach Zielgruppe waren unterschiedliche Faktoren wichtig für die optimale Qualität der Teamleistung. Fünf Prinzipien wurden dann allerdings für alle erfolgreichen Teams ausfindig gemacht:

1. Die gemeinsame Überzeugung der Teammitglieder, dass innerhalb der Gruppe ein „sicherer Raum“ gegeben ist, um auch zwischenmenschliche Risiken, wenn nötig, einzugehen.

2. Verlässlichkeit einer Leistung im vereinbarten Zeitrahmen und deren Qualität.

3. Ziele, Rollen und Abläufe strukturieren und klar formulieren.

4. Das sinnhafte Arbeiten, was auch den Teammitgliedern wichtig ist.

5. Der Einfluss des Teams auf die Arbeitsgestaltung selbst.

Hier wurde vor allem als Ergebnis deutlich, dass die von Edmondson (2019) beschriebene ,psychologische Sicherheit" der entscheidende Faktor für die erfolgreiche Zusammensetzung und -arbeit in einem Team ist (vgl. Duhigg 2017, S. 66; zur Metaanalyse vgl. Frazier et al. 2017).

Sollte dies auch Relevanz gewinnen können in Bezug auf Erfolgsaussichten von Post-Merger-Integrationsprozessen? Unstrittig ist, dass Teams und Teamarbeit im Rahmen der M\&A-Projekte eine grundlegende Säule des gesamten Prozesses sind und über die Qualität sowie die Ergebnisse von Teamarbeit hohe Bedeutung für das Gelingen von PMI-Prozessen gewinnen (vgl. Berner 2017, S. 212). Dabei sollte bereits im Vorfeld der PMI-Phase gehandelt werden. Dies betrifft zunächst einmal schon das „who“, also die Zusammensetzung von Teams als solchen, aber auch die Organisation der Teamarbeit und das „how“, also Methoden, Instrumente, Vorgehensbedingungen und Aufgabenverteilung von Teamarbeit. Besonders relevant ist dies in Bezug auf die sog. „Integrationsteams“ des Post-Merger-Teils des M\&A- 
Prozesses. Diese Teams tragen und realisieren wesentlich diese Vernetzungsphase, die in der Literatur z. T. auch explizit so bezeichnet wird (vgl. Jansen 2008, S. 249). Sie sollen die Integration realisieren und sind daher in der Regel aus Mitgliedern aller vormals selbstständigen Unternehmensparteien zusammengesetzt. Neuzusammensetzungen dieser Teams stehen in direktem Zusammenhang zu der Integrationsaufgabe im Kontext der weitreichenden Umstrukturierungen im Post-MergerIntegrationsprozess. Vor dem Hintergrund der ermittelten fünf Prinzipien ist eine Labilisierung vormals bestehender psychologischer Sicherheit mit Bezug auf die oben genannten Prinzipien zu erwarten.

Nur einige Aspekte können hierbei besonders hervorgehoben werden: Nach Einschätzung von Edmondson (2019, S. 16f.) ist Vertrauen von hoher Bedeutung für den Erfolg von Teams, um möglicherweise auftretende unterschiedliche Sichtweisen im Team zu respektieren und gemeinsam zu besprechen. Sonst besteht die Gefahr, dass Team-Mitglieder nicht mehr offen ihre Meinung äußern, Ideen einbringen und Ansichten sowie Entscheidungen anderer diskutieren können oder wollen. Dass dies in der heiklen Integration unterschiedlicher Werte, Befindlichkeiten, Situationslagen und Entscheidungserfordernisse der PMI von herausragender Bedeutung ist, wenn die Integrationsaufgabe bzw. die vom Integrationsteam zu bearbeitende Teilaufgabe hierzu gelingen soll, ist offenkundig. Sonst besteht die Gefahr, dass der Gesamtprozess durch suboptimale Entscheidungen und Folgehandlungen quasi ,,von unten her" zersetzt bzw. immer stärker zum Entgleisen gebracht wird. Dieser Effekt verstärkt sich, wenn sich das ,ungesicherte“ Teamklima durch zunehmendes Misstrauen weiter verhärtet und auf die Handlungsfähigkeit des Teams negativ auswirkt. Unter der Voraussetzung, dass solche destruktiven Teamprozesse dann sogar gleichzeitig in mehreren Integrationsteams entstehen und diese gegeneinander agieren, kann es zu Blockaden oder mindestens Verlangsamungen von Entscheidungsfindungen, Fehlentscheidungskaskaden und Rückbezugsschleifen kommen, die das PMI-Projekt insgesamt beeinträchtigen.

Die Themen und Aufgabenstellungen der PMI-Integration betreffen grundlegende strukturelle und prozessuale Veränderungen sowie Weichenstellungen für eine erfolgreiche, effektive und nachhaltige Gesamtintegration. Diese wird dann in den Teilaspekten erfolgreich, effektiv und nachhaltig gestört. Vor dem Hintergrund der angespannten Managementsituation mit einem auf schnelle Verbesserung von Unternehmensergebnissen ausgerichteten Management kann der Integrationsprozess auf diese Weise in eine ,bleierne Schwere“ geraten und möglicherweise sogar gefährdet werden. Ähnliche Folgen lassen sich auch in Bezug auf weitere von Edmondson entwickelte Prinzipien und Aspekte psychologischer Sicherheit bzw. deren Entgleisen in Teams aufzeigen, z. B. möglichst gleich verteilte Redeanteile, soziale Empathie und Sensibilität, Fehlertoleranz, Förderung von Neugier und Experimenten, Schätzen individueller Talente und Kompetenzen mit einer stärkenorientierten Perspektive sowie insbesondere ein wertschätzender und respektvoller Umgang miteinander.

Wenn psychologische Sicherheit eine Schlüsselbedeutung für die Entfaltung von Kreativität und Leistung hat, ist sie gerade in der PMI-Phase besonders wichtig, um etwa Teammitglieder zu ermuntern, sich ohne Furcht vor negativen Konsequenzen einzubringen - und damit ist sie letztlich die Voraussetzung für das Gelingen des PMI-Prozesses. Es dürfte einleuchten, dass dies mit einer allein betriebswirtschaft- 
lichen Betrachtungsart kaum angemessen reflektiert und gefördert werden kann, sondern dass es hierfür einer prozessbezogenen und psychosozial fokussierten Perspektive bedarf. Kompetenzen hierzu werden von Edmondson (2019, S. 153-183) aufgeführt, insbesondere: Fähigkeit zum Perspektivwechsel, Kommunikation auf Augenhöhe, Antizipieren von Reaktionen, eine explorative und dialogische Haltung. Diese müssen in den Prozess einbezogen werden, um die im Vergleich zu einer stabilen Unternehmenssituation exponentielle Erhöhung der psychosozialen Komplexität in PMI-Phasen angemessen einzubeziehen.

Dabei sind weitere Aspekte relevant, die in der psychosozialen Begleitung der PMI-Phase entsprechend berücksichtigt werden müssen: Je nachdem, ob Mitarbeitende z.B. auf der Seite des akquirierenden oder des übernommenen Unternehmensteils engagiert waren, könnten erhebliche emotionale Varianzen vorliegen, z. B. Gefühle wie Überlegenheit, Stärke, Dominanz, Sicherheit oder Niedergeschlagenheit, Schwäche, Enttäuschung, sich unterordnen zu müssen, Unsicherheit und Angst, möglicherweise auch Angst vor Kontrollverlust, Erleichterung, Ärger über zusätzliche Aufgabenbereiche, die in Formen des Widerstands münden. Es liegt auf der Hand, dass in dieser Umgebung nicht nur eine entsprechende Reflexivität, sondern vor allem eine psychosoziale Handlungsfähigkeit erforderlich und einzubringen ist, die psychodynamisch-systemische Perspektiven umfasst, und zwar nicht nur ergänzend, quasi als Nebenprozess, sondern im Kernvollzug der Beratung: prozessanalytisch sowie in Bezug auf die Handlungsinstrumentarien im Prozess. Dies gilt erst recht, insofern es in der PMI-Phase schon selbst zu einer deutlichen Abnahme der Leistung und des Sich-bekennens zum bisherigen Unternehmen kommen kann - mit Symptomen von Arbeitsunzufriedenheit oder ,,innerer Kündigung“ als emotionalen Reaktionen. Der Begriff „Merger-Syndrom“ bezeichnet dies einschlägig (Dickmann 2002, S. 124; zit. n. Barnikel 2007). Zu vermuten ist, dass Projektleitung und Berater/ innen-Stab im Falle einer Absorption des einen vormals selbstständigen Unternehmensteils in den anderen, also bei einem Merger of Non-Equals (der „Große schluckt

Tab. 1 The Leader's Tool Kit. (Edmondson 2019, S. 159)

\begin{tabular}{llll}
\hline Kategorie & Voraussetzungen schaffen & Einladung zur Teilnahme & Produktiv reagieren \\
\hline $\begin{array}{l}\text { Aufgaben } \\
\text { für }\end{array}$ & $\begin{array}{l}\text { Der Arbeit einen Bezugs- } \\
\text { rahmen geben }\end{array}$ & $\begin{array}{l}\text { Situations-bezogene Demut } \\
\text { zeigen }\end{array}$ & Wertschätzung ausdrücken \\
Führungs- & Erwartungen in Bezug auf & Schwächen zugeben & Zuhören \\
kräfte & Scheitern, Unsicherheit und & Proaktives Nachforschen & Anerkennung und Dank \\
& wechselseitige Abhängig- & praktizieren & Das Scheitern vom Stigma \\
& keit formulieren, um die & Gute Fragen stellen & befreien \\
& Notwendigkeit des Äußerns & Vorbild für intensives & Nach vorne schauen \\
& der eigenen Stimme zu & Zuhören sein & Hilfe anbieten \\
& betonen & Strukturen und Prozesse & Diskutieren, überlegen \\
& Die Sinnausrichtung beto- & einführen & und mit Brainstorming \\
& nen & Foren für Input schaffen & nächste Schritte finden \\
& Aufzeigen, was auf dem & Richtlinien für Diskussio- & Klare Verstöße mit Sank- \\
& Spiel steht, warum und für & nen erarbeiten & tionen belegen \\
& wen es wichtig ist & & \\
Was & Geteilte Erwartungen und & Die Überzeugung, dass die & Kontinuierliches Lernen \\
wird & Bedeutung & eigene Stimme willkom- & als Orientierung \\
damit & & men ist & \\
erreicht? & & & \\
\hline
\end{tabular}


den Kleinen"), in dieser Hinsicht besonders gefordert sind. Mit der Zurverfügungstellung von ,The Leader's Tool Kit“, also des Methodenkoffers der Führenden für den Aufbau von psychologischer Sicherheit, hat Edmondson (2019, S. 159) ihre Erfahrungen für die Praxis zugänglich gemacht (s. Tab. 1).

Dies sind sinnvolle Leitlinien, die allerdings noch genauer auf PMI-Prozesse bezogen werden müssen und dabei ein besonderes psychosoziales Kompetenzprofil voraussetzen, das nach der bisherigen Argumentation integraler Bestandteil der Beratungsarbeit in PMI-Prozessen ist.

\section{Blick in die Expertenpraxis: Beobachtungen und Tendenzen}

Der folgende Abschnitt bietet eine Vorabauswertung von Ergebnissen einer qualitativen Untersuchung, die Alexander Pfurr im ersten Quartal 2021 im Rahmen eines Forschungsprojekts an der Universität Kassel vorgenommen hat. Dabei wurden anhand eines teilstrukturierten Leitfadens acht meist einstündige Interviews geführt. Die Stichprobe besteht aus Experten im Top-Management, im M\&A Sektor, in der Post-Merger-Integration sowie in der Transformationsberatung. Erfahrungen der Experten liegen in verschiedenen Branchen sowie Unternehmensgrößen vor. Erkenntnisinteresse bzw. Forschungsfragen richteten sich darauf, ob und inwiefern Aspekte psychologischer Sicherheit als Thema bzw. Konzept in die Durchführung von Fusionsprozessen einbezogen und berücksichtigt wurden sowie welcher Stellenwert diesen Aspekten und Prinzipien psychologischer Sicherheit dabei zugemessen wird. Außerdem wurde exploriert, welches Interventionsinstrumentarium von den Experten eingesetzt wird, um Unsicherheit im PMI-Prozess zu containern und eine angstreduzierte Atmosphäre zu ermöglichen. Im Rahmen einer qualitativen Inhaltsanalyse und mit dem Einsatz eines Kategoriensystems wurden die gewonnenen Daten ausgewertet.

Als erstes und eindeutiges Ergebnis lässt sich festhalten: Alle acht befragten Experten setzten in den von ihnen betreuten PMI-Projekten keine spezifisch strukturierten, systematischen Vorgehensweisen ein, mit denen Aspekte und Prinzipien der psychologischen Sicherheit bewusst und zielgerichtet aufgebaut werden sollten. Die Perspektive der psychologischen Sicherheit wurde auf der Ebene des Designs ihrer Fusionsprozesse entsprechend nicht berücksichtigt. Alle acht Befragten teilten zudem mit, dass ihnen das Konzept der psychologischen Sicherheit im Sinne von Edmondson als psychosozial bezogenes Theorienkonzept bisher nicht explizit bekannt ist, also als Erfolgsperspektive von PMI-Prozessen auf konzeptioneller Ebene auch gar nicht einbezogen werden kann. Die allgemeinen Hinweise zur Handlungsrelevanz durch den „Methodenkoffer“ kommen entsprechend (noch) nicht zur Anwendung, geschweige denn daraus abgeleitete Handlungskonzepte oder -konsequenzen für die Anlage von Post-Merger-Prozessen.

Aufgrund der oft kurzen Dauer der akuten Phase einer PMI wird von den Befragten unterschiedlich eingeschätzt, ob das Konzept der psychologischen Sicherheit überhaupt (positive) Wirkung entfalten könnte. Hierzu äußerten sich zwei der Befragten kritisch. Geteilte Meinungen liegen vor im Hinblick auf Praktikabilität, Notwendigkeit und Erkenntnis- und Handlungsgewinn eines derartigen Konzepts; 
zwei der Befragten hielten es für fragwürdig, eine angstfreie Organisation als Leitziel überhaupt zu propagieren. Alle acht Befragten sehen allerdings sehr wohl die Bedeutung der Ängste der Mitarbeitenden und einer konstruktiven Dynamik für den Erfolg bzw. Misserfolg der Post-Merger-Integration. Alle Befragten halten es für erforderlich, diese so früh wie möglich zu berücksichtigen und zu überwinden. Es zeigt sich, dass die befragten Experten zwar die Bedeutung von in der Konzeption von Edmondson entwickelten Aspekten und Wirkprinzipien der psychologischen Sicherheit kennen und die Wichtigkeit von psychologischer Sicherheit als solcher bestätigen, aber nicht konzeptionell reflektiert vorgehen. De facto werden im PMI-Prozess allerdings implizit sehr wohl Interventionen gesetzt und Aktivitäten entwickelt, die sich mit Prinzipien und Handlungsempfehlungen der Konzeption Edmondsons decken.

Die Befragungen ergaben, dass sich die Definition von Erfolg im Zusammenhang mit der Post-Merger-Integration unterscheidet: Erfolg wird von zwei der Befragten vor allem mit Zahlen, Daten, Fakten zur Unternehmensperformance und dem Aktienkurs im eher kurzfristigen Sinn bestimmt. Die Mehrheit der Befragten beschrieb eine langfristige Perspektive von Erfolg als maßgeblich. Weniger als 50\% der Integrationen werden von den Experten in diesen Kategorien als erfolgreich angesehen. Das Gestalten einer sicheren Arbeitsatmosphäre wird von allen Befragten bei der Auswahl von fünf vorgelegten Faktoren als wichtigstes Erfolgskriterium für PMI-Prozesse genannt. Das Schaffen von gemeinsamen Arbeitsabläufen erhielt die zweitmeisten Punkte gefolgt von der Festlegung von gemeinsamen IT-Standards, der Etablierung einer innovativen und kreativen Kultur und dem Aufbau von Organisationsstrukturen. Das obige paradoxe Ergebnis wird somit bestätigt: Aspekte psychologischer Sicherheit erhalten eine hohe Bewertung, das konkrete wissenschaftliche Konzept selbst ist aber unbekannt und wird von den Expertenpraktikern prognostisch eher als nicht unbedingt notwendig erachtet.

Die Befragung ergab weiterhin durch mehrfache Nennungen sieben Faktoren, die Scheitern bzw. Misserfolg einer Fusion unwahrscheinlicher machen:

1. Eine strategische Geschäftslogik. Dazu gehört die Auswahl eines realistischen Übernahmeziels verbunden mit einer frühzeitigen Besprechung und Anleitung der Integrationsarbeit im Rahmen der Due-Diligence-Phase.

2. Eine sensible Führung. Die Führungsspitze muss den Post-Merger-Prozess initiieren und unterstützen, so früh wie möglich. Die oberste Führungskraft hat die Pflicht, involviert zu sein und die Leitung zur Chefsache zu machen. Dabei ist die Befähigung zur Selbstkritik und (wenn nötig) zur Handlungskorrektur zu erwähnen. Idealerweise kommt es zu einer vertrauensvollen Zusammenarbeit und Unterstützung des Managements durch die Eigentümervertreter.

3. Die Unternehmenskultur. Dabei wird anerkannt, dass Veränderungen Zeit brauchen und schrittweise erfolgen müssen, in einer Umgebung, die durch Offenheit für die Kultur und die Eigenheiten des anderen gekennzeichnet ist. Nur durch einen respektvollen und toleranten Umgang ist nach Ansicht der Befragten die Grundlage für ein gemeinsames Vorankommen gelegt.

4. Eine emotional ansprechenden Vision. Eine solche Vision sollte erreichbar und realistisch sein. Die klare und kontinuierliche Kommunikation einer solchen Zielvorstellung schafft erst die enge Bindung zu den Mitarbeitenden. 
5. Das Projektmanagement wird oft als nicht stark genug beschrieben und als vernachlässigt angesehen, was auch durch den Einsatz von externen Beratern korrigiert werden könne.

6. Der Wille und das Engagement von allen Beteiligten, also nicht nur der Führungskräfte, insbesondere der Mitarbeitenden, ist mitentscheidend für den Erfolg. Die Unterstützung muss willentlich vorliegen. Aktives Mitgestalten ergibt sich als Folge des Verstehens der Ziele (emotionales Mittragen einer solchen Fusion).

7. Die Bindung der Mitarbeitenden. Insbesondere bei Fusionen zwischen Dienstleistungsunternehmen sind das unbedingte Einbinden und die Motivation der Verantwortungsträger wichtig, besonders bei Bankenmergern. Hierbei wird es als erfolgsfördernd erlebt, wenn es gelingt, die Mitarbeitenden aus den unterschiedlichen Kulturen so sensibel, verständnis- und respektvoll zu behandeln, dass eine neue Gemeinschaft zwischen den Kolleg/innen entstehen kann.

Diese sieben Faktoren zur Vermeidung des Scheiterns von Unternehmensfusionen bestätigen und erweitern in Bezug auf die Bedeutung der psychologischen Sicherheit das bisher gewonnene Bild: Sie lassen sich als Prinzipien der psychologischen Sicherheit im Sinne von Edmondson verstehen. Psychologische Sicherheit erweist sich so ex negativo als bedeutender Erfolgshorizont von PMI-Prozessen für die Befragten. Dies gilt auch für Handlungskonzepte, die aufgrund solcher Analysen von den Befragten für erforderlich gehalten werden, um Erfolgsfaktoren zu implementieren und Bedingungen für den Fusionserfolg zu fördern. Im Kern geht es dabei um Rahmenbedingungen für ,sichere Atmosphären“, wie sie auch Edmondson (2019, S. 182) in ihrem Konzept der psychologischen Sicherheit als hoch bedeutsam einschätzt - allerdings ohne explizite Bezugnahme auf die Konzeption Edmondsons durch die Befragten.

Dabei benannten die Befragten Interventionen, die in drei Kategorien zusammengefasst wurden, um eine entsprechende sichere Atmosphäre zu schaffen bzw. die fusionierte Organisation psychologisch ,,sicherer“ zu machen: Teamdimensionierung, Teambuilding und Teamarbeit sowie Führungsarbeit.

(1) Teamdimensionierung betrifft die Teambesetzung und zeigt sich in zwei Interventionen:

1. Einen Rahmen setzen für die Arbeit im Team sehen mehrere der Befragten als Handlungsperspektive an. Hier sind die konkreten Erwartungen an das Team zuerst zu formulieren und vom Top-Management zu kommunizieren. Ein Rahmen, also eine Umgebung mit Grenzen für die gemeinsame Arbeit, wird gesetzt, in dem sich das System der Teammitglieder entfalten kann. Die Unterschiedlichkeit der Teammitglieder selbst bzw. deren Herkunft treten so idealerweise in den Hintergrund gegenüber der Aufgabenerfüllung, Neuausrichtung, Einbringen von Ideen sowie Kreativität, was in den Fokus rückt. Letztlich geht es um eine Transformation der angstbesetzten Situation in eine Best-Practice-Dynamik, die sich aus der Klarheit der Aufgabenstellung ergeben kann. Der Change wird angelegt durch das Bewusstmachen der Verantwortung für die Zukunft. Dabei ist eine fachliche Kompetenz über das nötige Prozesswissen im Team sicherzustellen. 
2. Die Zusammensetzung der Teams sehen alle Befragten als wichtigen Ansatzpunkt an. Auswahl und Benennung der Teammitglieder von Integrationsteams erfolgen im Anschluss an die oben beschriebene Rahmensetzung durch das Top-Management sowie die Besetzung der Leitungsposition des Integrationsteams. Die zu bestimmenden Mitglieder des Teams sollen aus beiden vormals selbstständigen Unternehmensparteien bestellt werden, idealerweise paritätisch und gleichberechtigt auf Augenhöhe. Einige der Befragten empfehlen, einen kleineren Kreis (bis zu 5 Personen) zu benennen. Dabei ist eine diverse Auswahl der Kandidaten in Hinblick auf Kultur, Alter und Genderkriterien mit einer ganzheitlichen und prozessualen Sicht auf das Unternehmensgeschehen mit hoher Qualität und Fachkompetenz sicherzustellen. Vor allem muss eine positive Grundeinstellung zur Fusion gewährleistet sein. Über einen Lenkungskreis sollte auch regelmäßig die Linienführung, die das reguläre Geschäft zu verantworten hat, eingebunden werden. Durch die paritätische Besetzung des Teams kann die Zusammenarbeit schneller gelingen und kann eher eine harmonische und vertrauensvolle Atmosphäre entstehen, in der dann auch Grundhaltungen für eine angstreduzierte Organisation entwickelt werden können. Als sinnvolles Werkzeug wird von einem der Befragten eine vorausgehende „Stakeholder Analyse“ genannt, mit der die Einflussnahme, die Bereitschaft und das Interesse der Kandidat/innen für die Integrationsteams an der erfolgreichen Gestaltung der Fusion untersucht werden soll. Dabei wird zuerst das Profil festgelegt, dann gesucht und wenn möglich über das Ausrichten von Assessment Centern die optimale Passung ermittelt. Damit wird auch das Finden und die Bindung von unbedingt zu haltenden Leistungsträgern unterstützt.

(2) Teambuilding und Teamarbeit folgt als zweite Kategorie. Für die Mehrheit der Befragten stellt sie die wichtigste Interventionsperspektive dar, insofern als die meisten Interventionen im Prozess hier angesetzt werden, um eine „neue Gemeinschaft" zu formen bzw. zu fördern. Hierzu gehören kulturbildende Maßnahmen, humorvolle Begleitung, Förderung von Kommunikationsbereitschaft in den Integrationsteams, in der oberen Führungsmannschaft, aber auch in der Organisation insgesamt, um elitäre Abspaltungen zu vermeiden. Als Tool wird von einem der Befragten der Einsatz von externen Begleitern zur Unterstützung der Leitenden als Impulsgeber/innen und Moderatoren dringend empfohlen, um das Management vom Umsetzungsdruck zu entlasten. Die Ergebnisse der Befragung lassen sich wiederum in fünf Unterkategorien systematisieren:

1. Fairness und gute Sitten im Umgang miteinander zu fördern, nannten alle Befragten. Durch Interventionen wie Coaching-Angeboten für Mitarbeitende werden etwa Transparenz, Offenheit und Vertrautheit hergestellt und eine positive Haltung unterstützt. Seriosität und Anständigkeit durch Integrität unterstützen die Motivation der verbleibenden Mitarbeitenden zusätzlich. Hierzu gehört auch ein wertschätzender Umgang ,auf Augenhöhe“, um Angst und Ungewissheit zu reduzieren und Kreativität und Einsatzfreude zu stärken.

2. Aktives Zuhören, persönlich-dialogischer Kontakt und explizite Rückmeldekultur wurden von allen Befragten genannt. Dies verlangt die Präsenz der Führenden oder bei örtlicher Trennung den Einsatz von digitalen Medien. Die Haltung, die 
im ganzen Unternehmen etabliert werden sollte, ist durch das Prinzip der „Offenen Tür", also durch einen einfachen Zugang zu Führungskräften gekennzeichnet, durch Erreichbarkeit, Wertschätzung, Respekt und authentisches Interesse sowie Zeit für Gespräche ohne Arroganz.

3. Beziehung aufbauen: Über situativ angepasste Maßnahmen wird persönliches Kennlernen zwischen den Teammitgliedern ermöglicht. Das authentische „Care“ mit wirklichem Interesse am anderen legt den Grundstein des Beziehungsaufbaus. Unterstützt werden kann dies durch moderierte Übungen und Vorstellungsaktivitäten mit dem Ziel einer Vernetzung zwecks sozialem Austausch. Hierbei scheint den Befragten wichtig, allen einen Raum zu geben und darauf zu achten, dass alle gleiche Anteile am Geschehen haben. Ein Tool, der hier wichtig ist, ist auch „Abschied zu feiern“: Mit einem Ritual wird das in der Vergangenheit Erlebte gemeinsam gefeiert, aber dann mit einem Endpunkt abgeschlossen. So kann ein emotionales Loslassen des Vergangenen erfolgen, um den Weg in das Neue freizumachen. Auf Vorschlag eines Interviewten erscheint es sinnvoll, die neue Leitung „von außen“ zu besetzen, um nicht eine der beiden vormals getrennten Gruppen zu bevorzugen, sondern eine neue Gemeinschaft zu bilden, an der sich die bisher nicht geeinten Kolleg/innen gemeinsam ,abarbeiten“ können.

4. Direkte Ansprache und Motivation: Eine klare Linie und Ansage durch die Leitung fördert eine Best-of-Leistungskultur ohne Bevorzugung einer Seite. Ein gleichberechtigter Umgang und das Zurückstellen von wertenden Äußerungen muss nicht nur propagiert, sondern auch wirklich gelebt werden. Dabei sind Konflikte ernst zu nehmen und in zeitnahen Interventionen anzugehen. Das sachlich gehaltene Gespräch, gebündelt mit einer Ansprache in Wir-Form durch die Leitung, fördert das Gefühl der Zusammengehörigkeit und reduziert damit die Angst im Umgang miteinander.

5. Die Beteiligung von Mitarbeitenden: Durch das offene und direkte Involvieren der Mitarbeitenden in den Teams und der Leistungsorganisation wird das erfolgreiche Zusammenwachsen ermöglicht und gefördert. Die Interventionen konzentrieren sich dabei darauf, ressourcen- und kompetenzbasiert an Lösungen der Zukunftsherausforderungen zu arbeiten. Wichtig ist dabei, die Erfahrungen der Mitarbeitenden aufzunehmen und einzubeziehen, insbesondere derjenigen Mitarbeitenden, die die Arbeit bisher geleistet haben.

(3) Führungsarbeit: Die dritte Kategorie wird mit Führungsarbeit zusammengefasst, wobei sich die konkreten Interventionen und Handlungen der acht Befragten in neun Unterkategorien systematisch darstellen lassen. Von allen Interviewpartnern wird gesehen, dass die jeweilige Führungsphilosophie und -kultur den Rahmen für die konkreten Aktivitäten steckt und dabei den Handlungsspielraum für die beratende Begleitung setzt:

1. Führungspositionen: Eine zentrale Bedeutung kommt nach Einschätzung von sechs Befragten der Besetzung von Führungspositionen zu. Sie sollte aus beiden Unternehmensteilen erfolgen, sofern gleiches Potenzial und gleiche Leistung vorliegen. Der Einsatz von externen Experten mit Kompetenzen für Auswahl und Bewertung der Kandidat/innen führt zu einer breiteren Akzeptanz bei den Mit- 
arbeitenden. Hinsichtlich des Kompetenzprofils von Führungskräften sollte eine Balance zwischen Theorie und Praxis gewährleistet sein.

2. Angstbewältigung: Hohe Bedeutung in der Wahrnehmung von Führungsaufgaben kommt nach Einschätzung von allen Interviewten der Aufgabe zu, Angst zu nehmen. Ob psychologische Sicherheit entstehen kann, ist in hohem Maße abhängig vom Verhalten der involvierten Führungskräfte auf allen Führungsebenen, vom Top-Level bis zur operativen Leitung. Als Grundhaltung muss der respektvolle Umgang mit allen Beteiligten gelten. Wenn die Führungskraft auf dieser Grundlage persönlich bei Verfehlungen eingreift und den Prozess gestaltet, fördert dies die Bindung von Wissensträgern an das Unternehmen. Einen offenen und ungezwungenen Zugang zur Führungskraft sicherzustellen, trägt dazu bei, Ängste zu nehmen.

3. Change: Für drei Befragte ist es wichtig, dass die Führung explizit den Fokus auf Change und den Veränderungsprozess setzt. Change bedeutet, die Menschen in der Organisation jeweils in (neue) Verantwortung zu bringen. Mitarbeitende sollen befähigt werden, zum einen die eigenen Kompetenzen und Stärken zu erkennen, zu bündeln und dafür die nötigen Aushandlungsräume zu schaffen. Zum anderen geht es um die Ausrichtung des Prozesses auf die Zukunft - im Gegensatz zu ständiger Rückbesinnung auf das Vergangene. Der Fokus soll dabei nicht auf die Unterschiedlichkeiten der zu Fusionierenden gelegt werden, sondern auf einen Umgang mit Verschiedenheit, die als Ressource gesehen und genutzt wird. Die Post-Merger-Integration zeigt sich auf diese Weise als Transformation zu etwas Besserem und Neuem.

4. Ziele definieren: Dies ist für alle Experten grundlegend. Mit konkreten Zielvorgaben wird den Teams Orientierung gegeben. Dabei muss möglichst von Anfang an klar sein, was erwartet wird. Dies wird ausgedrückt in quantitativen und qualitativen Zielvorgaben. Dabei gilt die folgende Erfolgsformel: Ziel setzen, Ziel verständlich machen und Zielerreichung unterstützen.

5. Planen: Auch das Planen ist eine entscheidende Führungsaufgabe im Post-Merger-Prozess. Das Etablieren von professionellem Projektmanagement steht im Zentrum der Interventionen. Dabei ist ein konsequenter, aber nicht starrer Plan $\mathrm{zu}$ entwickeln, der genügend Freiräume lässt, damit von den Mitarbeitenden gemeinsam sinnvolle Handlungsoptionen in Richtung der gesetzten Ziele entwickelt werden können. Daraus sind Arbeitspakete abzuleiten und zu vereinbaren. Gemischte Teams (s. oben) sollten selbstbestimmt und in Eigeninitiative an der Umsetzung arbeiten, sodass psychologische Sicherheit entstehen kann.

6. Delegieren und Organisieren: Die Top-Führung muss durch Delegation, Organisation und Korrektur, wenn nötig, regelmäßig intervenieren. Dabei kommt es immer auf die Situation der jeweiligen Fusion, der Art und Ausrichtung an. Immer ist allerdings ein systematischer Aufbau aller Handlungen zu berücksichtigen und entsprechend zu organisieren. Mit Sorgfalt ist die Arbeit und sind die Entscheidungsbefugnisse an die Mitarbeitenden zu verteilen. Überwachen und Gegensteuern bei Abweichungen im gesetzten Zeitrahmen bleibt aber der Führung überlassen. Mit dem Tool „Workshops“, also geplante Sitzungen abzuhalten, steht ein Mittel der gezielten Ansprache zur Verfügung, um die Arbeitsbeziehung zu etablieren und Raum zu geben für das klärende Gespräch mit Fragen und Antworten. 
7. Vorbildfunktion: Führungskräfte haben für alle Befragten eine Vorbildfunktion. Es gilt, nicht nur darüber zu sprechen, sondern auf der Grundlage einer persönlichen Integrität ins Handeln zu kommen. Eine Politik der „offenen Tür“ ist hilfreich (s. oben), vor allem aber das wirkliche Vorleben der definierten Werte. Führungskräfte sollen sich dabei nicht „,verstecken“, sondern sichtbar sein und der Fusion Aufmerksamkeit schenken. Eine solche Vorbildfunktion ist vom gesamten Führungsgremium einheitlich vorzuleben. Widersprüche bzw. widersprüchliche Handlungen sind zu vermeiden, weil Inkonsistenz Verwirrung stiftet und Unsicherheit entsteht.

8. Konsequentes Handeln: Wichtig ist hier, konsequent $\mathrm{zu}$ handeln und ggf. auch Konsequenzen zu ziehen. Wer im Unternehmen bleibt und wer nicht, muss möglichst zu Beginn des Integrationsprozesses verbindlich festgelegt werden. Mit dieser Klärung bzw. der damit verbundenen Entlassung bzw. Trennung von Personen, die nicht mitziehen wollen und womöglich (nicht konstruktiven) Widerstand leisten, wird ein unmissverständlicher Impuls an die im Unternehmen Verbleibenden geschickt, was eine befreiende Wirkung im Team entfalten kann. So wird auch psychologische Sicherheit der Mitarbeitenden und Engagierten gestärkt und deren Angst reduziert, auf allen Hierarchieebenen im Unternehmen, inklusive TopManagement.

9. Kommunikation: Alle Experten unterstreichen die Bedeutung der Kommunikation durch die Führungskräfte. Gut bedachte Interventionen im Rahmen einer offenen und ehrlichen Kommunikation auf allen Ebenen der Unternehmensorganisation können eine stabilisierende Bedeutung für den PMI-Prozess gewinnen. Dies vermittelt Glaubwürdigkeit und einen Umgang mit der Wahrheit nach bestem Wissen und Gewissen. Verwirrung und Fake News sind auch in Bezug auf die Presse- und Öffentlichkeitsarbeit auf alle Fälle zu vermeiden. Erfolge sind zu kommunizieren sowie gebührend zu feiern und tragen zur Bildung des Zusammengehörigkeitsgefühls bei.

\section{Ausblick und Empfehlungen}

Die Zusammenstellung der Befragungsergebnisse zeigt klare Tendenzen und auch gemeinsame Einschätzungen der Befragten in Hinsicht auf Bedeutung und Ausgestaltung von Interventionen und Interventionskategorien im Post-Merger-Integrationsprozess. Für die noch im Pionierstatus befindliche weitere wissenschaftliche Durchdringung der PMI-Prozesse liegt bald ein Grundlagenmanual vor, mit dessen Hilfe in den einzelnen Kategorien und Unterkategorien die Bedeutung für das Gelingen der Integrationsphase weiter untersucht werden kann. Angesichts der erheblichen betriebs- und volkswirtschaftlichen Kosten des Scheiterns solcher Prozesse ist weitere Empirie dringend angeraten.

Auch wenn die befragten Experten die Konzeption der psychologischen Sicherheit von Edmondson nicht kannten und nicht in ihre Prozessgestaltungen einbringen konnten, deutet vieles darauf hin, dass die psychologische Sicherheit für die von den Befragten genannten Kategorien und Unterkategorien in der PMI-Phase eine Schlüsselbedeutung hat. Dies genauer zu untersuchen und dabei die von Edmondson ermit- 
telten Aspekte und Prinzipien zu berücksichtigen, soll die Handlungssicherheit von Akteuren in der PMI-Phase forschungsgestützt erhöhen und eine Reflexionsbasis für Analysen und Handlungskonzepte, aber auch für Prognosen über Erfolgsaussichten von PMI-Prozessen schaffen. Besonders wichtig ist dabei das Profil und die Professionalität von Berater/innen. Schon die Einschätzungen der befragten Experten machen deutlich, dass eine rein betriebswirtschaftliche Perspektive von Beratung unzureichend ist und dass reflexive Beratungskompetenzen in der PMI-Phase wesentlich und integral psychosoziale Kompetenzen umfassen müssen, um den Prozess konstruktiv zu begleiten.

Ein weiterer Aspekt der Auswertung betrifft die genauere Kategorisierung solcher Fusionsprozesse. Integrationsprozesse im Rahmen von Fusionen können je nach Kontext, Ausrichtung und Größe von Unternehmen und Organisationen große Differenzen der erforderlichen Prozessgestaltung und Umsetzungen aufweisen. Situation und spezifische Profile von Fusionslagen bedingen, dass die Interventionen und Maßnahmen entsprechend anzupassen sind. Auch hier gilt es, weiter zu forschen und ggf. Profile von Fusionslagen und -herausforderungen genauer in den Blick zu nehmen, um sie hinsichtlich der notwendigen Handlungsweisen $\mathrm{zu}$ ordnen und zu klassifizieren. Ein Blick auf die Integrationskarte im folgenden Schaubild zeigt die Wirkung der psychologischen Sicherheit in unterschiedlichen Integrationssituationen (s. Abb. 1).

An dieses Schema gilt es anzuknüpfen, es ist zu verfeinern und als Grundlage einer Handlungskasuistik weiterzuführen. Insofern stellt psychologische Sicherheit eine heuristische Basis für die PMI-Prozesse dar. Rein funktionale Logiken, einseitig egoistische Interessen von Akteuren (z. B. des Top-Managements) sowie ungenügende oder fehlende Kommunikation führen im PMI-Prozess zu Unsicherheit, Disharmonie, zum Gefühl von Ohnmacht und Ausgeliefertsein - und damit schließlich zu Ängsten. Solche Ängste veranlassen die Mitarbeitenden, sich abzukoppeln oder oft sich aus Unternehmen zu verabschieden - mit der Folge, dass Wissen und Kreativität

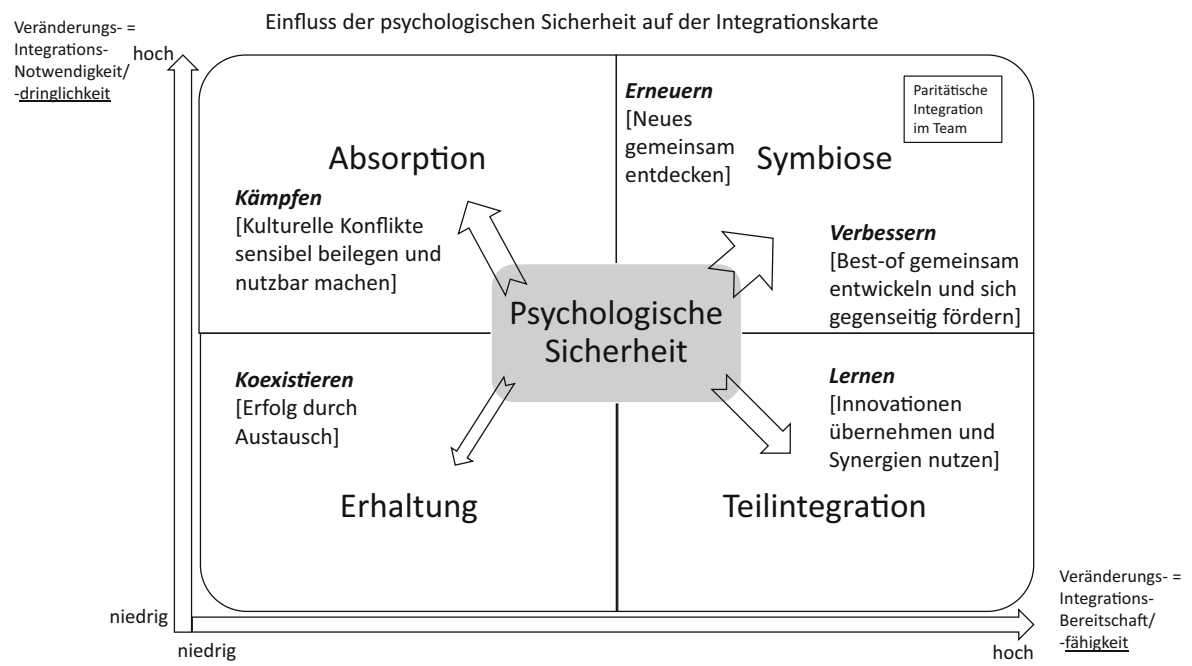

Abb. 1 Einfluss der psychologischen Sicherheit auf der Integrationskarte 
verloren gehen und PMI-Prozesse scheitern. Eine situationsgerechte und kontinuierliche Anlage psychologischer Sicherheit ist deshalb auch in betriebswirtschaftlicher Hinsicht fundamental.

Externe Berater kommen nach Einschätzung der befragten Experten in weniger als der Hälfte der Prozesse zum Einsatz - meistens erst dann, wenn bereits Probleme entstanden sind. Angesichts der konstitutiven Bedeutung der psychologischen Sicherheit ist der Einsatz externer Berater in PMI-Prozessen dann sinnvoll, wenn die Beratungskompetenz neben betriebswirtschaftlicher Expertise auch psychosoziale Kompetenzen umfasst, die wissenschaftlich ausgewiesen sind. Letzteres ist auf Basis der Befragungsergebnisse auch aus betriebswirtschaftlicher Perspektive zu fordern. Denn die in den PMI-Prozessen erforderliche Komplexität ist von betriebswirtschaftlicher Expertise allein nicht zu leisten und in der Regel auch nicht vom Management allein, das im PMI-Prozess stark unter dem Druck von Stakeholdern steht. Aufgrund dieser Drucksituation bedarf es einer auf psychosoziale Prozessberatung ausgerichteten und zugleich betriebswirtschaftlich bezogenen Beratungsperspektive.

Funding Open Access funding enabled and organized by Projekt DEAL.

Open Access Dieser Artikel wird unter der Creative Commons Namensnennung 4.0 International Lizenz veröffentlicht, welche die Nutzung, Vervielfältigung, Bearbeitung, Verbreitung und Wiedergabe in jeglichem Medium und Format erlaubt, sofern Sie den/die ursprünglichen Autor(en) und die Quelle ordnungsgemäß nennen, einen Link zur Creative Commons Lizenz beifügen und angeben, ob Änderungen vorgenommen wurden.

Die in diesem Artikel enthaltenen Bilder und sonstiges Drittmaterial unterliegen ebenfalls der genannten Creative Commons Lizenz, sofern sich aus der Abbildungslegende nichts anderes ergibt. Sofern das betreffende Material nicht unter der genannten Creative Commons Lizenz steht und die betreffende Handlung nicht nach gesetzlichen Vorschriften erlaubt ist, ist für die oben aufgeführten Weiterverwendungen des Materials die Einwilligung des jeweiligen Rechteinhabers einzuholen.

Weitere Details zur Lizenz entnehmen Sie bitte der Lizenzinformation auf http://creativecommons.org/ licenses/by/4.0/deed.de.

\section{Literatur}

Backovic, L., Flauger, J., Kolf, F., Obmann, C., \& Scheppe, M. (2019). Fusionen: Warum Übernahmen so oft scheitern. Handelsblatt, 16.2.2019. www.handelsblatt.com. Zugegriffen: 1. Apr. 2021.

Barnikel, K. (2007). Post-Merger Integration. München: Salzwasser.

Berner, W. (2017). Systemische Post-Merger-Integration. Stuttgart: Schäfer Poeschel.

Coenenberg, A. G., Schultze, W., \& Biberacher, J. (2002). Akquisition und Unternehmensbewertung. In W. Busse von Colbe, et al. (Hrsg.), Betriebswirtschaft für Führungskräfte. Stuttgart: Schäffer-Poeschel.

Duhigg, C. (2017). Smarter, faster, better. The transformative power of real productivity. New York: Random House.

Edmondson, A.C. (2019). The fearless organization: creating psychological safety in the workplace for learning, innovation, and growth. Hoboken: Wiley.

Frazier, M. L., Fainshmidt, S., Klinger, R. L., Pezeshkan, A., \& Vracheva, V. (2017). Psychological safety: a meta-analytic review and extension. Personnel Psychology, 70(1), 113-165.

Jansen, S. A. (2008). Mergers \& Acquisitions: Unternehmensakquisitionen und -kooperationen - Eine strategische, organisatorische und kapitalmarkttheoretische Einfïhrung (5. Aufl.). Wiesbaden: Gabler. 


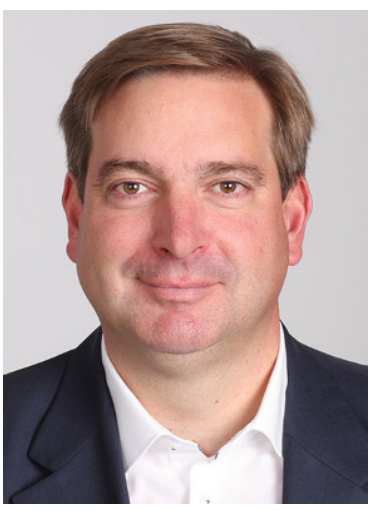

Dipl.-Oec. Alexander Pfurr DipM MBA, Studium der Wirtschaftswissenschaften, Marketing, Management, Coaching, Organisationsberatung und Supervision an den Universitäten Kassel und Bradford/GB. Bis 2021 international erfahrene Führungskraft und Geschäftsführer im thyssenkrupp Konzern. Seit 2018 Berater für Unternehmen, M\&A und Corporate Finance sowie Coach für Führungskräfte, Teams und Organisationen. Gründer der ISABEA consult UG. www.acxit.com und www.ella-executives.de.

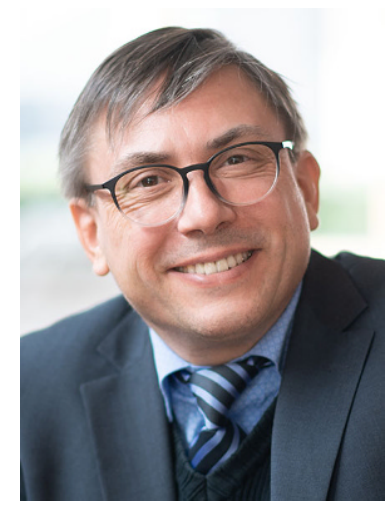

Prof. Dr. Freimut Schirrmacher M.A., Studium der Ev. Theologie, Pädagogik, Philosophie, Sozialmanagement, Coaching, Organisationsberatung und Supervision. Zertifizierte Weiterbildungen in Psychotherapie, Mediation sowie Innovations- und Transformationsmanagement. Promotion in Systematischer Theologie/ Religionsphilosophie. Habilitation in Praktischer Theologie an der Kirchlichen Hochschule Wuppertal/Bethel. 1998-2015 Wiss. Assistent und Pfarrdienst, 2015-2021 Direktor des Ev. Fröbelseminars der Diakonie Hessen, seit 2021 Pfarrer bei der Bundesbereitschaftspolizeidirektion. Langjährige Beratungstätigkeit, seit 2018 Beratung von Führungskräften, Teams und Organisationen. 